\title{
STRONGLY BRANCHED COVERINGS OF CLOSED RIEMANN SURFACES ${ }^{1}$
}

\author{
ROBERT D. M. ACCOLA
}

\begin{abstract}
Let $b: W_{1} \rightarrow W_{2}$ be a $B$-sheeted covering of closed Riemann surfaces of genera $p_{1}$ and $p_{2}$ respectively. $b$ is said to be strongly branched if $p_{1}>B^{2} p_{2}+(B-1)^{2}$. If $M_{2}$ is the function field on $W_{1}$ obtained by lifting the field from $W_{2}$ to $W_{1}$, then $M_{2}$ is said to be a strongly branched subfield if the same condition holds.

If $M_{1}$ admits a strongly branched subfield, then there is a unique maximal one. If $M_{2}$ is this unique one and $f$ is a function in $M_{1}$ so that

$$
(B-1) o(f)<\left(p_{1}-B p_{2}\right)+(B-1)
$$

then $f \in M_{2}$, where $o(f)$ is the order of $f$. (This is a generalization of the hyperelliptic situation.) These results are applied to groups of automorphisms of $W_{1}$ to obtain another generalization of the hyperelliptic case.
\end{abstract}

I. Introduction. The main purpose of this paper is to generalize the following properties of hyperelliptic Riemann surfaces. Let $W$ be a hyperelliptic Riemann surface of genus $p(p \geqq 2)$ with hyperelliptic conformal self-map $T$. Let $b: W \rightarrow W /\langle T\rangle$ be the analytic map from $W$ onto the orbit space of $\langle T\rangle$, where $W /\langle T\rangle$ is a Riemann surface of genus zero. Then $\langle T\rangle$ is normal in the full group, $A(W)$, of conformal self-maps of $W$. Moreover, the quotient group, $A(W) /\langle T\rangle$, is isomorphic to a finite subgroup of $A(W /\langle T\rangle)$. Finally, if $f$ is a meromorphic function on $W$ of order no greater than $p$, then $f$ is the lift, via the projection $b$, of a rational function on $W /\langle T\rangle$.

Let $b: W_{1} \rightarrow W_{2}$ be an analytic map of closed Riemann surfaces of $B$ sheets and total ramification $r_{b}$. If $p_{i}$ is the genus of $W_{i}$ for $i=1,2$ then the Riemann-Hurwitz formula reads

$$
2 p_{1}-2=B\left(2 p_{2}-2\right)+r_{b} \text {. }
$$

Presented to the Society, November 22, 1967; received by the editors January 13, 1970.

AMS 1969 subject classifications. Primary 3045, 1245; Secondary 1240.

Key words and phrases. Riemann surface, coverings of closed Riemann surfaces, function fields, linear series, automorphism, strongly branched coverings, strongly branched subfields.

1 This paper appeared in Notices Amer. Math. Soc 15 (1968), p. 238. While working on this paper the author received support from several sources. (1) Research partially sponsored by the Air Force Office of Scientific Research, Office of Aerospace Research, United States Air Force, under AFOSR grant No. AF-AFOSR-1199-67. (2) National Science Foundation grant GP-7651. (3) Institute for Advanced Study grant-in-aid. 
The map $b$ will be called strongly branched if

$$
r_{b}>2 B(B-1)\left(p_{2}+1\right) \text {. }
$$

Notice that for $B=2, p_{2}=0$, and $r_{b}>4$ we are in the hyperelliptic case, for then $p_{1}>1$. The aspect of the hyperelliptic situation we are stressing then is that for fixed $p_{2}$ and $B, r_{b}$ is large. Note that the identity map is not strongly branched.

A conformal self-map of a Riemann surface will be called an automorphism. The full group of automorphisms of a surface $W$ will be denoted $A(W)$. Our hypotheses will always imply that the genus of $W$ is at least two and so $A(W)$ will be a finite group. If $G$ is a subgroup of $A(W)$ then the space of orbits of $G, W / G$, is naturally a Riemann surface so that the projection $W \rightarrow W / G$ is an analytic map and the number of sheets is equal to the order of $G$. One of the generalizations of the hyperelliptic case, Corollary 3, is as follows. If $G$ is a simple group and the map $W \rightarrow W / G$ is strongly branched then $G$ is normal in $A(W)$.

Again let $b: W_{1} \rightarrow W_{2}$ be an analytic map which is not necessarily strongly branched. Continue the notation of the previous paragraph. Let $f_{1}$ be a meromorphic function on $W_{1}$ of order $o\left(f_{1}\right)$. Let $f_{1}^{1}, f_{1}^{2}, \cdots$, $f_{1}^{B}$ be the branches of $f_{1}$ on the $B$ sheets of the covering. Define $D_{b}\left(f_{1}\right)$ as follows.

$$
D_{b}\left(f_{1}\right)=\prod_{i<j}\left(f_{1}^{i}-f_{1}^{j}\right)^{2} \cdot{ }^{2}
$$

Then $D_{b}\left(f_{1}\right)$ is a well-defined function on $W_{2}$ whose order is at most $2(B-1) o\left(f_{1}\right)$. The number of zeros of $D_{b}\left(f_{1}\right)$ is at least $r_{b}{ }^{3}$ Thus if $r_{b}>2(B-1) o\left(f_{1}\right)$ then $D_{b}\left(f_{1}\right) \equiv 0$. The following lemma follows from well-known principles.

Lemma $1 .{ }^{4}$ If $r_{b}>2(B-1) o\left(f_{1}\right)$ then the map $b: W_{1} \rightarrow W_{2}$ admits $a$ factoring $c: W_{1} \rightarrow W_{3}$ and $d: W_{3} \rightarrow W_{2}$ where $b=d \circ c, c$ is not one-to-one, and there is a function $f_{3}$ on $W_{3}$ so that $f_{1}=f_{3} \circ c$.

The definition of strongly branched is designed to assure that the hypothesis of Lemma 1 holds for functions on $W_{1}$ whose order is at most $B\left(p_{2}+1\right)$. Notice that if $B$ is a prime integer then the map $d$ of

${ }^{2} D_{b}\left(f_{1}\right)$ is usually called the discriminant. See Hensel-Landsberg [3, p. 26].

${ }^{3}$ If the poles of $f_{1}$ lie on branch points of $b$ this need not be correct. Rather than make a more complicated computation it is better to replace $f_{1}$ by $T f_{1}$ where $T$ is a fractional linear transformation. The results will then hold for $T f_{1}$. But the nature of all results of this paper is that they will then hold for $f_{1}$.

4 The author first observed this technique in Röhrl [4]. 
Lemma 1 must be one-to-one. For composite $B$, however, a nontrivial factorization may occur. In this latter case it would appear that the intermediate surface, $W_{3}$, depends on the choice of $f_{1}$. The main result of this paper, Lemma 4 , states that a $W_{3}$ can be chosen independently of any $f_{3}$ satisfying the hypotheses of Lemma 1. Moreover, $W_{3}$ can be chosen uniquely.

The methods of this paper give fairly simple proofs of some wellknown results. We include proofs of some classical results on linear series, the usual proofs being somewhat inaccessible. We also give constructive examples of closed surfaces of genus five or more which admit only the identity automorphism. ${ }^{5}$

The results of this paper can be expressed in terms of function fields, and we will find it convenient to do so. If $b: W_{1} \rightarrow W_{2}$ is a $B$ sheeted covering, let $M_{1}$ be the field of meromorphic functions on $W_{1}$ and let $M_{2}$ be that field in $M_{1}$ obtained by lifting all functions from $W_{2}$ to $W_{1}$ via $b$. The index of $M_{2}$ in $M_{1}$ is $B$. Then there is a one-to-one correspondence between nonconstant subfields of $M_{1}$ and surfaces of the type $W_{2}$. Moreover, if fields are ordered by inclusion and surfaces are ordered by the obvious covering requirement then this correspondence preserves these partial orderings. Consequently, we will call a subfield $M_{2}$ a strongly branched subfield if the covering $W_{1} \rightarrow W_{2}$ is strongly branched.

Using the Riemann-Hurwitz formula (1) and the definition of strongly branched (2) we obtain other criteria for strongly branched which are listed in the next lemma. Criterion (i) allows one to define the concept without reference to the ramification. Criterion (ii) is useful in the proofs.

LEMMA 2. If $b: W_{1} \rightarrow W_{2}$ is a covering of closed surfaces then the following conditions are equivalent to $b$ being strongly branched:

(i) $p_{1}>B^{2} p_{2}+(B-1)^{2}{ }^{6}$

(ii) $B r_{b}>(B-1)\left(2 p_{1}-2+4 B\right)$.

II. Some classical results. We now use the ideas introduced in $\$ I$ to prove the classical results mentioned earlier.

Proposition I. Let $W_{1}$ be a Riemann surface of genus $p_{1}$. Let $f$ and $g$ be two meromorphic functions on $W_{1}$ of orders $F$ and $G$ respectively. If $f, g$ generate the full field of functions on $W_{1}$ then

${ }^{5}$ The proof that there are surfaces of every genus $(p \geqq 3)$ with trivial automorphism groups will be found in Baily [1].

${ }^{6}$ The consequences of criterion (i) with $p_{2}=0$ are discussed by Dorodnov [2]. For $\mathrm{B}$ prime part of the results of this paper are included in his. 


$$
p_{1} \leqq(F-1)(G-1) .^{7}
$$

Proof. Consider $f: W_{1} \rightarrow W_{2}$ where $W_{2}$ is the Riemann sphere and $r_{f}$ is the total ramification. Since the field generated by $f$ and $g$ separates points of $W_{1}$, it follows that $D_{f}(g)$ cannot be identically zero. ${ }^{8}$ Thus $r_{f} \leqq 2(F-1) G$. But $2 p_{1}-2=-2 F+r_{f}$. The result follows immediately. q.e.d.

Proposition II. Let $W_{1}$ be a Riemann surface of genus $p_{1}$. Let the constant function $1, f$, and $g$ be three linearly independent meromorphic functions on $W_{1}$ so that $f$ and $g$ have the same order $F$. Suppose further that the poles of $f$ and $g$ are simple and they coincide. If $f$ and $g$ generate the field of functions on $W_{1}$ then

$$
p_{1} \leqq \frac{1}{2}(F-1)(F-2) \cdot{ }^{9}
$$

Proof. The proof is the same as that of Proposition I except that the added information about the poles of $f$ and $g$ allows one to deduce that $o\left(D_{f}(g)\right) \leqq F(F-1)$, and so $r_{f} \leqq F(F-1)$. q.e.d.

Propositions I and II can be interpreted to give conditions when two functions do not generate the function field on $W_{1}$.

Corollary 1. Assume all hypotheses of Proposition I except the last. Assume that $p_{1}>(F-1)(G-1)$. Then $f$ and $g$ generate a strongly branched subfield.

Corollary 2. Assume all hypotheses of Proposition II except the last. Assume that $p_{1}>\frac{1}{2}(F-1)(F-2)$. Then $f$ and $g$ generate a strongly branched subfield.

Proof. Since the proofs of the corollaries are almost identical we include only a proof of Corollary 2. Let $M_{2}$ be the field generated by $f, g$ and the constants and let $M_{1}$ be the full field on $W_{1}$. Let the $B$ sheeted covering $b: W_{1} \rightarrow W_{2}$ correspond to the inclusion of fields

${ }^{7}$ See Hensel-Landsberg [3, p. 385]. The method of proof presented here is essentially that of Hensel-Landsberg. It will be understood that the field generated by $f$ and $\boldsymbol{g}$ will include the constant field, $\boldsymbol{C}$.

${ }^{8}$ We may again assume that no pole of $g$ lies on a branch point of $f$.

- The hypotheses of Proposition II make most sense in the context of linear series. Let $D$ be the polar divisor of $f$. If we consider those divisors equivalent to $D$ which are parametrized by the linear family generated by $f, g$ and the constant function 1 , we obtain a linear series of dimension 2 and order $F$, denoted $g_{F}^{2}$. To say that " $f, g$ and 1 generate $M_{1}$ " is rendered in the usual terminology by " $g_{F}^{2}$ is simple." Here the triple $(f, g, 1)$ gives a birational map of $W_{1}$ onto a plane curve of order $F$. Proposition II is simply the fact that the genus of a plane algebraic curve of order $F$ is bounded by $\frac{1}{2}(F-1)(F-2)$. See Hensel-Landsberg [3, p. 427] or Walker [5, p. 179]. 
$M_{2} \subset M_{1}$. Let $p_{2}$ be the genus of $W_{2} . f$ and $g$ arise from functions on $W_{2}$ of order $F / B$. By Proposition II

$$
p_{2} \leqq \frac{1}{2}(F / B-1)(F / B-2),
$$

so

$$
B^{2} p_{2}+(B-1)^{2} \leqq \frac{1}{2}(F-B)(F-2 B)+(B-1)^{2} .
$$

The corollary will follow by criterion (i) of Lemma 2 if

$$
\frac{1}{2}(F-B)(F-2 B)+(B-1)^{2} \leqq \frac{1}{2}(F-1)(F-2) .
$$

That is $0 \leqq 3 B F-3 F-4 B^{2}+4 B$ or $0 \leqq(B-1)(3 F-4 B)$.

Since $1, f$, and $g$ are linearly independent,

$$
2 \leqq B \leqq F / 2 \text {. }
$$

The result now follows. q.e.d.

That strongly branched coverings arise in this context perhaps indicates that the concept is a little less artificial than the original ad hoc definition might indicate.

III. Main results. We shall now adopt the following notational convention. Analytic maps will be denoted by small letters $b, c, \cdots$; the number of sheets denoted by the corresponding capital letters: $B, C, \cdots$; and total ramification denoted: $r_{b}, r_{c}, \cdots$. Surfaces $W_{i}$ will have genus $p_{i}$ for $i=1,2, \cdots$. All surfaces will be covered by $W_{1}$. The function field on $W_{1}$ will be denoted by $M_{1}$. If $b: W_{1} \rightarrow W_{i}$ then $M_{i}\left(\subset M_{1}\right)$ will be the function field of index $B$ obtained by lifting functions from $W_{i}$ to $W_{1}$ via $b, i=2,3, \cdots$.

Lemma 3. Let $b: W_{1} \rightarrow W_{2}, c: W_{1} \rightarrow W_{3}, d: W_{3} \rightarrow W_{2}$ be analytic maps such that $b=d \circ c$. If $b$ is strongly branched then either $c$ or $d$ is.

Proof. Assume that neither $c$ nor $d$ is strongly branched. Then $p_{1} \leqq C^{2} p_{3}+(C-1)^{2}$ and $p_{3} \leqq D^{2} p_{2}+(D-1)^{2}$. Therefore

$$
p_{1} \leqq(C D)^{2} p_{2}+C^{2}(D-1)^{2}+(C-1)^{2} .
$$

Since $B=C D$ it suffices to show that

$$
C^{2}(D-1)^{2}+(C-1)^{2} \leqq(C D-1)^{2}
$$

in order that $b$ be not strongly branched. This is straightforward. q.e.d.

Definition. A strongly branched subfield $M_{2}$ of $M_{1}$ will be called a maximal strongly branched subfield of $M_{1}$ if whenever $M_{2} \subset M_{3} \subset M_{1}$, $M_{2} \neq M_{3}$ then $M_{3}$ is not a strongly branched subfield. The corresponding definition will also hold for coverings. 
LEMMA 4. Let $b: W_{1} \rightarrow W_{2}$ be a maximal strongly branched covering. Suppose $f_{1}$ is a funciion on $W_{1}$ so that $2(B-1) o\left(f_{1}\right)<r_{b}$.

Then there is an $f_{2}$ on $W_{2}$ so that $f_{1}=f_{2} \circ b$ (i.e., $\left.f_{1} \in M_{2}\right)$.

Proof. ${ }^{10}$ Lemma 1 assures us of a factorization $c: W_{1} \rightarrow W_{3}, d: W_{3}$ $\rightarrow W_{2}$ where $b=d \circ c, c$ is not one-to-one, and there is an $f_{3}$ on $W_{3}$ so that $f_{1}=f_{3} \circ c$. Choose $W_{3}$ so that $D$ is minimum. If $D>1$ we will show that $2(D-1) o\left(f_{3}\right)<r_{d}$. Another application of Lemma 1 will give a factorization of $d$ and a contradiction of the minimality of $D$. Then the proof will be complete.

So assume $D>1$. Now $b$ is strongly branched but $c$ is not. Consequently $d$ is strongly branched. This means

$$
r_{c} \leqq 2 C(C-1)\left(p_{3}+1\right)
$$

and

$$
r_{d}>\frac{D-1}{D}\left(2 p_{3}-2+4 D\right)
$$

Thus $r_{c} \leqq C(C-1)\left(2 p_{3}-2+4 D\right)-4 C(C-1)(D-1)$ or

$$
r_{c}<\frac{C D(C-1)}{(D-1)} r_{d}
$$

Several applications of the Riemann-Hurwitz formula give $r_{b}=r_{c}$ $+C r_{d}$ which together with (6) and the fact that $B=C D$ yields $r_{b}<(C+B(C-1) /(D-1)) r_{d}$ or

$$
(D-1) r_{b}<C(B-1) r_{d} .
$$

Since $\operatorname{Co}\left(f_{3}\right)=o\left(f_{1}\right)$ we have

$$
2(D-1) o\left(f_{3}\right)=\frac{2(D-1)}{C} o\left(f_{1}\right)<\frac{(D-1)}{C(B-1)} r_{b}<r_{d} . \quad \text { q.e.d. }
$$

LEMMA 5. If a maximal strongly branched subfield of $M_{1}$ exists, then it is unique.

Proof. Suppose $b: W_{1} \rightarrow W_{2}$ and $c: W_{1} \rightarrow W_{3}$ are two maximal strongly branched coverings. We may assume that $B\left(p_{2}+1\right)$ $\geqq C\left(p_{3}+1\right)$. Let $f_{3}$ be a function on $W_{3}$ of order no greater than $p_{3}+1$. Then $f_{3} \circ c$ is in $M_{3}$ and has order no greater than $C\left(p_{3}+1\right)$. Thus, by Lemma $4, f_{3} \circ c \in M_{2}$. Since $M_{3}$ is generated by functions of order

${ }_{10}$ The author is indebted to W. T. Kiley for showing how the original version of this paper could be considerably simplified. In particular, the idea of the proof of Lemma 4 is due to him. 
$C\left(p_{3}+1\right)$ or less, we have $M_{3} \subset M_{2} \subset M_{1}$. Since $M_{3}$ is maximal it follows that $M_{3}=M_{2}$. q.e.d.

We will summarize the above results in the following theorem.

THEOREM 1. Let $M_{1}$ be the field of functions on a Riemann surface of genus $p_{1}$. Suppose $M_{1}$ admits a strongly branched subfield. Then there exists a unique maximal strongly branched subfield $M_{2}$ of genus $p_{2}$ and index $B$. All strongly branched subfields lie in $M_{2}$. If $f_{1}$ is a function in $M_{1}$ so that either

(i) $(B-1) o\left(f_{1}\right)<\left(p_{1}-B p_{2}\right)+(B-1)$ or

(ii) $o\left(f_{1}\right) \leqq B\left(p_{2}+1\right)$,

then $f_{1} \in M_{2}$.

PROOF. If $M_{1}$ admits a strongly branched subfield $M_{3}$ then there is a maximal strongly branched subfield $M_{2}$ so that $M_{3} \subset M_{2} \subset M_{1}$. Since $M_{2}$ is unique the first two assertions of the theorem are proved. Note that (ii) implies (i) and that (i) is just Lemma 4 with $r_{a}$ replaced using the Riemann-Hurwitz formula. q.e.d.

IV. Applications to automorphisms of Riemann surfaces. Let $W_{1}$ be a Riemann surface whose function field, $M_{1}$, admits a maximal strongly branched subfield $M_{2}$. Let $W_{1} \rightarrow W_{2}$ be the corresponding covering. Any automorphism of $W_{1}$, acting on $M_{1}$, leaves $M_{2}$ invariant, since $M_{2}$ is unique. Let $N$ be the subgroup of $A\left(W_{1}\right)$ which leaves the functions of $M_{2}$ pointwise fixed. Then $N$ is easily seen to be a normal subgroup of $A\left(W_{1}\right)$ and is, in fact, the group of cover transformations $^{11}$ of the cover $W_{1} \rightarrow W_{2}$. The quotient group $A\left(W_{1}\right) / N$ is isomorphic to a subgroup of $A\left(W_{2}\right)$. Moreover, this subgroup of $A\left(W_{2}\right)$ must respect the branching of the covering $W_{1} \rightarrow W_{2}$ in the obvious manner. Finally, if $M_{1}$ is a Galois extension of $M_{2}$ then $N$ is the corresponding Galois group and $N$ has order equal to the index of $M_{2}$ in $M_{1}$. We summarize this discussion in the following theorem which can be viewed as a generalization of the hyperelliptic case.

THEOREM 2. Suppose $W_{1}$ admits a group of automorphisms whose fixed field is a strongly branched subfield. Then $M_{1}$ is a Galois extension of the maximal strongly branched subfield whose Galois group is normal in $A\left(W_{1}\right)$.

COROLlARY 3. If $W_{1}$ admits a simple group of automorphisms whose fixed field is strongly branched, then this group is normal in $A\left(W_{1}\right)$.

${ }^{11}$ In the context of a branched covering $b: W_{1} \rightarrow W_{2}$ a cover transformation $T$ is an automorphism of $W_{1}$ such that $b \circ T=b$. 
Proofs. If $M_{3}$ is a strongly branched subfield and $M_{1}$ is a Galois extension of $M_{3}$ then $M_{1}$ is a Galois extension of the maximal strongly branched subfield, $M_{2}$, since $M_{3} \subset M_{2} \subset M_{1}$. If the Galois group of $M_{1}$ over $M_{3}$ is simple then $M_{2}=M_{3}$ since otherwise the simple group would contain a nontrivial normal subgroup. q.e.d.

The above discussion can also be used to construct Riemann surfaces which admit only the identity as an automorphism. Let $W_{2}$ be a Riemann surface and let $T$ be a finite set on $W_{2}$ so that the only automorphism of $W_{2}$ which permutes $T$ is the identity. Now let $b: W_{1} \rightarrow W_{2}$ be a strongly branched covering branched only over $T$ and such that the only cover transformation is the identity. Then in the discussion preceding Theorem $2, N$ and $A\left(W_{1}\right) / N$ must both be the identity, and so also then is $A\left(W_{1}\right)$. To be more specific, let $p_{2}=0$, $B=3$ and $r_{b}>12$. Then the covering $b$ will be strongly branched. To insure that $N=\{$ id $\}$ it suffices to have at least one branch point of multiplicity 2 since the only candidate for the Galois group is $Z_{3}$. Choose the points $T$ so that no fractional linear transformation permutes them. Since the order of $T$ is more than 4 this can be achieved. The surfaces thus constructed will have genus $p_{1}>4$.

\section{REFERENCES}

1. Walter L. Baily, Jr., On the automorphism group of a generic curve of genus $>2$, J. Math. Kyoto Univ. 1 (1961/2), 101-108; correction, p. 325. MR $26 \# 121$.

2. A. V. Dorodnov, On the structure of fields of algebraic functions, Kazan State Univ. Sci Survey Conf., 1962, Izdat. Kazan Univ., Kazan, 1963, pp. 21-22. (Russian) MR 32 \#5649.

3. K. Hensel and G. Landsberg, Theorie der algebraischen Funktionen einer Variablen und ihrer Anwendung auf algebraische Kurven und Abelsche Integrale, Chelsea, New York, 1965. MR 33 \#272.

4. Helmut Röhrl, Unbounded covering of Riemann surfaces and extensions of rings of meromorphic functions, Trans. Amer. Math. Soc. 107 (1963), 320-346. MR 26 \#6397.

5. Robert Walker, Algebraic curves, Dover, New York, 1962. MR 26 \#2438.

Brown University, Providence, Rhode Island 02912 